\title{
Spine Curve Measures Taken in Individuals with Osteoporosis and Osteopenia Using the IONmed Mobile Phone Application Are Highly Consistent with Digital Inclinometer Measures
}

\author{
Norma J. MacIntyre, ${ }^{1}$ Chris P. Recknor, ${ }^{2}$ and Julie C. Recknor ${ }^{2}$ \\ ${ }^{1}$ School of Rehabilitation Science, McMaster University, IAHS 403, 1400 Main Street West, Hamilton, ON, Canada L8S 1C7 \\ ${ }^{2}$ United Osteoporosis Centres, 2350 Limestone Parkway, N.E., Gainesville, GA 30501, USA \\ Correspondence should be addressed to Norma J. MacIntyre; macint@mcmaster.ca
}

Received 30 October 2013; Accepted 8 December 2013; Published 17 April 2014

Academic Editors: Y.-W. Hsieh, C.-L. Kao, and A. Ozcan Edeer

Copyright (C) 2014 Norma J. MacIntyre et al. This is an open access article distributed under the Creative Commons Attribution License, which permits unrestricted use, distribution, and reproduction in any medium, provided the original work is properly cited.

\begin{abstract}
Introduction. This study determined the agreement between measures of standing posture in individuals at risk of osteoporotic fracture obtained using the IONmed mobile phone application and a digital inclinometer. Methods. One experienced rater assessed 20 adults, aged 55-88 years, attending an outpatient osteoporosis clinic. A standardized protocol was duplicated to acquire a single measure of spine curvature (lumbosacral angle, lordosis, kyphosis) using the IONmed mobile phone application and a digital inclinometer. Interdevice agreement was determined using the intraclass correlation coefficient (ICC) with 95\% confidence intervals (CI) and standard error of the measurement (SEM) with 95\% CI and Bland-Altman plots. Results. ICC (95\% CI) for lumbosacral angle, lordosis, and kyphosis is $0.97(0.92,0.99), 0.97(0.92,0.99)$, and $0.99(0.98,1.0)$, respectively. SEM (95\% CI) for lumbosacral angle, lordosis, and kyphosis is $0.99^{\circ}(0.75,1.44), 1.33^{\circ}(1.01,1.94)$, and $1.23^{\circ}(0.94,1.80)$, respectively. Measurement bias is small. Conclusions. In our sample, the IONmed mobile phone application provides measures highly consistent with the digital inclinometer measures of standing posture. This method offers clinicians a quick, convenient way of identifying alterations in spine alignment which require followup and remediation.
\end{abstract}

\section{Introduction}

Thoracic kyphosis increases with age such that kyphotic angle progresses by $3^{\circ}$ per decade in men and women over the age of 50 years [1]. Exact prevalence of excessive kyphosis (hyperkyphosis) is difficult to determine due to the lack of a gold standard for diagnosis. Regardless of how it is measured, hyperkyphosis is common in older adults and of particular concern for people with osteoporosis [2]. In a prospective study of 1624 postmenopausal women with osteoporosis followed for three years, Roux and colleagues [3] found that the women in the highest tertile for measures of thoracic kyphosis at baseline were most likely to have suffered a vertebral compression fracture at followup even after adjusting for known risk factors. These findings confirm that hyperkyphosis is an independent risk factor for osteoporotic fractures of the spine. Bruno and colleagues [4] have investigated the compression loading on individual vertebral bodies (T6 to L5) resulting from body weight and muscle loading acting in the axial direction at incremental angles of thoracic hyperkyphosis $\left(50^{\circ}\right.$ to $\left.75^{\circ}\right)$ and considered two postural compensation strategies people use to maintain a stable centre of mass and horizontal eye gaze (one of which balances the thoracic and lumbar curvatures). The largest increases in vertebral body compression loading were observed in the thoracolumbar and lumbar regions, particularly in conditions where the spine curvatures were not balanced [4]. Recently, we reported that kyphotic angle is associated with self-reported activities of daily living (ADLs) and performance on a balance test in 36 patients attending an osteoporosis clinic. In our sample of high functioning older adults, $50 \%$ were hyperkyphotic according to normative 
values for digital inclinometer measures whereas only three participants had morphometric vertebral fractures [5]. These findings confirm previous observations that age-related hyperkyphosis develops due to a variety of reasons, only one of which is osteoporotic vertebral fracture. Back extensor muscle weakness is a common cause that is modifiable $[6,7]$. Postural training including exercises to improve back extensor muscle endurance or strength have improved kyphosis and physical performance [7] and reduced the incidence of vertebral compression fracture [8]. Therefore, it is important to measure standing posture in order to identify hyperkyphosis and associated compensations in the pelvis and lumbar spine indicative of the need for followup to address the associated impairments and activity limitations in older adults.

Several noninvasive methods have been developed for measuring sagittal plane spine curvatures in standing. Previously, the first author's group assessed the reliability of the flexicurve ruler and the digital inclinometer measures of spine curvatures taken by the same rater and determined that minimal gains in reliability are achieved when performing more than one trial using the digital inclinometer versus a minimum of three measures using the flexicurve ruler [9]. Moreover, test-retest reliability of a single measure of lumbosacral angle, lumbothoracic angle, and thoracocervical angle taken by an experienced rater using the digital inclinometer was very high in older adults attending a rheumatology clinic specializing in osteoporosis care [5]. The factory-calibrated digital inclinometer is relatively inexpensive ( $\$ 500$ USD) and it only takes a few minutes to acquire and manually record the measurements. Therefore, this method was recommended to identify hyperkyphosis and monitor the effectiveness of targeted exercises and education to improve or maintain standing posture [5]. Since the time when that study was conducted, Dr. C. P. Recknor (internist and second author) developed the IONmed mobile phone application for standing posture assessment in order to facilitate the inclusion of this measurement in the clinical setting. This application utilizes the same measurement protocol as that used for the digital inclinometer in combination with an internal calibrated accelerometer system in the mobile phone. The software, which can be downloaded onto a mobile phone that uses either an Android or an Apple operating system, guides the user through the steps to acquire measures of standing posture which are displayed on the device and automatically recorded to a database file. It is expected that the measured spine curvature angles are consistent with those acquired using the digital inclinometer. Therefore, the purpose of this study was to determine the agreement between measures of standing posture obtained using the IONmed mobile phone application designed to assess sagittal spine curvatures in individuals at risk of osteoporotic fracture and a digital inclinometer, previously shown to have excellent test-retest reliability in this population [5].

\section{Methods}

2.1. Design. Testing was completed during a single visit at an outpatient osteoporosis clinic. All measures were collected by the same rater with seven years of experience using a digital inclinometer to assess standing posture in patients attending the clinic. A single measure of the lumbosacral angle (S), standing lumbar posture (L), and standing thoracic posture $(\mathrm{T})$ was taken using the mobile phone application and duplicated immediately using the digital inclinometer following the same standardized procedure. The measurement protocol described in the Saunder's Digital Inclinometer User's Guide was followed. After removing their shoes and any bulky clothing, participants were instructed to stand erect and maintain their best posture throughout the procedure. The following three landmarks were palpated and the angle was measured: (1) at the sacral midpoint; (2) at the T12L1 interspace; and (3) at the C7-T1 interspace. The sacral midpoint and the T12-L1 interspace were accessed through light clothing whereas the C7-T1 interspace was exposed. The study protocol was approved by the Northeast Georgia Health System Institutional Review Board. All participants provided written informed consent prior to participating.

2.2. Participants. Patients attending the outpatient osteoporosis clinic between March 2013 and May 2013 were invited to volunteer during their visit in response to a study advertisement posted in the waiting room.

\subsection{Spine Curvature Measurements}

2.3.1. Mobile Phone Application. The IONmed mobile phone application was downloaded onto an iPhone 5 from https:// itunes.apple.com/us/app/spinecurve/id633986164? $1 \mathrm{~s}=1 \& \mathrm{mt}=$ 8. (Note that this application also is available for download to mobile phones using the Android operating system at: https://www.ionmed.us/ionmed/android.html.) The application was opened and the onscreen directions were followed. First, the flat surface of the top of the phone was placed against a flat vertical surface as indicated by an arrow on the mobile phone screen. The rater pressed the onscreen prompt to set the pitch to zero degrees. The rater placed the point of the arrow displayed on the mobile phone screen at the sacral midpoint and pressed the labelled bar to automatically record $S$ and reset that angle to zero degrees. The point of the arrow displayed on the mobile phone screen was then placed at the T12-L1 interspace and the labelled bar was pressed to automatically record $\mathrm{L}$ and reset that angle to zero degrees. Finally, the point of the arrow displayed on the mobile phone screen was placed at the C7-T1 interspace and the labelled bar was pressed to automatically record $\mathrm{T}$.

2.3.2. Digital Inclinometer. The digital inclinometer (Saunder's digital inclinometer, Empi Therapy Solutions) was placed against a flat vertical surface and the angle output was set to zero degrees. The inclinometer was initially placed at the sacral midpoint and the angle displayed on the digital screen was recorded as $\mathrm{S}$. After recording the angle, the rater pressed the reset button to set that angle to zero degrees. The inclinometer was then positioned at the T12-L1 interspace. The rater recorded the angle displayed on the digital screen as $\mathrm{L}$ and pressed the reset button to zero the inclinometer at this 
TABLE 1: Mean (SD) spine curvature angles measured, in degrees, using 2 different devices in 20 older adults standing with their best posture.

\begin{tabular}{lcc}
\hline & $\begin{array}{c}\text { IONmed mobile } \\
\text { phone application }\end{array}$ & $\begin{array}{c}\text { Digital } \\
\text { inclinometer }\end{array}$ \\
\hline Lumbosacral angle & $16.2^{\circ}(5.2)$ & $16.6^{\circ}(6.2)$ \\
Lumbar & $32.3^{\circ}(7.2)$ & $32.8^{\circ}(7.7)$ \\
Thoracic & $53.3^{\circ}(12.9)$ & $53.2^{\circ}(12.9)$ \\
\hline
\end{tabular}

angle. Finally, the rater positioned the inclinometer at the C7$\mathrm{T} 1$ interspace and recorded the angle displayed on the digital screen as $\mathrm{T}$.

2.4. Statistical Analysis. All statistical analyses were performed using SPSS release 20.0 software (SPSS Inc., Chicago, IL). We tested interdevice reliability of measures of $S$, L, and $\mathrm{T}$ with type 2,1 intraclass correlation coefficients (ICCs) [10], and the standard error of the measurement (SEM) which was calculated as the square root of the error variance [11]. Acceptable reliability was determined on the basis of a high ICC in combination with a low SEM. We also used BlandAltman plots as this is the recommended statistical method for assessing the agreement between any two test methods that purport to measure the same property [12].

\section{Results}

The participants ( $n=20,18$ women) were all Caucasian with an average age (SD) of 72.1 (9.8) years. One participant had osteopenia and 19 were diagnosed with osteoporosis, 11 of whom had a history of osteoporotic fracture. Table 1 summarizes the spine curve measures for this sample acquired using each of the two devices.

Interdevice reliability is high for all spine curve measures. ICC ( $95 \% \mathrm{CI})$ for S, L, and T is $0.97(0.92,0.99), 0.97(0.92$, $0.99)$, and $0.99(0.98,1.0)$, respectively. The SEM $(95 \% \mathrm{CI})$ for $\mathrm{S}, \mathrm{L}$, and $\mathrm{T}$ is $0.99^{\circ}(0.75,1.44), 1.33^{\circ}(1.01,1.94)$, and $1.23^{\circ}(0.94$, $1.80)$, respectively.

The Bland-Altman plots shown in Figures 1(a)-1(c) illustrate the small measurement bias (varying from $0.1^{\circ}$ for $\mathrm{T}$ to $-0.55^{\circ}$ for $\mathrm{L}$ ). The largest limits of agreement are observed for $L\left(-4.3^{\circ}, 3.2^{\circ}\right)$. Figure 1 confirms that no systematic differences in the spine angles measured using the two devices are observed as a function of the mean values.

\section{Discussion}

The purpose of this study was to determine the agreement between measures of standing posture obtained with a userfriendly mobile phone application designed to assess spine curvatures in people with low bone mass and a digital inclinometer, previously shown to have excellent test-retest reliability [5]. For our sample, excellent agreement between the measures of spine curvatures in standing was demonstrated by the high ICCs ( $\geq 0.97$ with lower $95 \%$ confidence bound $\geq 0.92$ ) and the low SEM. For example, we are $95 \%$ confident that the "true" value for $\mathrm{T}$ is somewhere between $0.94^{\circ}$ lower and $1.8^{\circ}$ higher than the single measured value obtained using either device. Bland-Altman plots confirm this with biases well within $1^{\circ}$ and limits of agreement $\leq$ $7.5^{\circ}$. There are no apparent systematic errors in the measures obtained using the two devices. This work confirms that the dedicated mobile phone application offers an alternative method for measuring standing posture in older individuals with osteoporosis that is quick and convenient for those healthcare providers who already use information technology to facilitate their clinical practice.

According to the Saunder's Digital Inclinometer User's Guide, normal thoracic standing posture (kyphosis) is between $30^{\circ}$ and $40^{\circ}$. Figure 1 (c) confirms that only three participants in the current study had a "normal" kyphotic standing posture. There is no gold standard for diagnosing hyperkyphosis; however, $40^{\circ}$ is also used as the threshold for classification based on the modified Cobb angle measured on standing lateral spine X-rays [13]. In this method, a goniometer is used to measure the angle at the intersection of lines drawn perpendicular to a line parallel to the superior border of T3 (or the most superior vertebral body border that can be observed) and a line drawn parallel to the inferior border of T12 identified on the radiograph [13]. In the Rancho Bernardo Study cohort of 553 men and 854 women 50 years of age or older recruited from the defined Southern California community, the majority of those over the age of 60 years had kyphotic angles (measured using the modified Cobb angle method) above $40^{\circ}$ and mean values increased with age, particularly among those with osteoporotic vertebral fractures [14]. For the group of women 70 to 79 years, the mean kyphotic angle was $51^{\circ}$ [14] which is comparable to the average value of $53^{\circ}$ for our study sample having an average age of 72 years. The reliability and validity of measures of kyphosis obtained using two common nonradiologic methods, the flexicurve ruler and the Debrunner kyphometer, have been established through comparison with the modified Cobb angle [15]. Previously, generalizability theory was applied to show that single measures obtained using the digital inclinometer are highly reliable in postmenopausal women with one or more osteoporotic vertebral fractures [9]. Subsequently, MacIntyre et al. confirmed that test-retest reliability of single digital inclinometer measures of spine curvatures was high $\left(\operatorname{ICC}(\operatorname{SEM})=0.91\left(2.5^{\circ}\right), 0.90\left(2.9^{\circ}\right)\right.$, and $0.91\left(3.5^{\circ}\right)$ for S, L, and T, resp.) [5]. Furthermore, measures of the lumbar lordosis and lumbosacral angle are obtained using this method thus providing important information about postural compensations employed by a person with hyperkyphosis to maintain a stable centre of mass and horizontal eye gaze shown to impact spine loads and fracture risk [4]. Thus, measures of kyphotic angle alone (Debrunner kyphometer) or kyphotic and lordotic indices only (flexicurve ruler) are likely to be inadequate in determining functional restrictions and fracture risk. It can be seen that the level of agreement between the measures obtained using two different devices in the current study is less than the error associated with duplicate measures taken using the digital inclinometer approximately nine days apart 


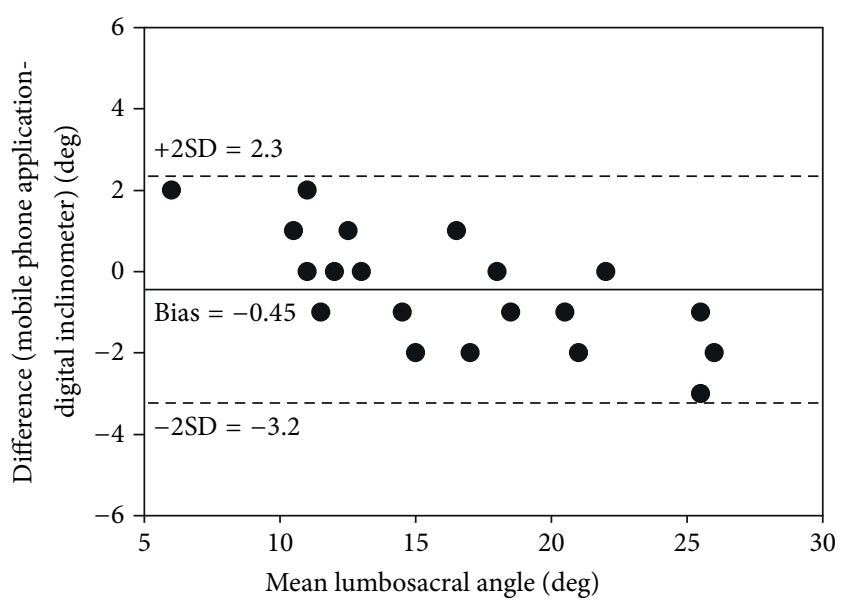

(a)

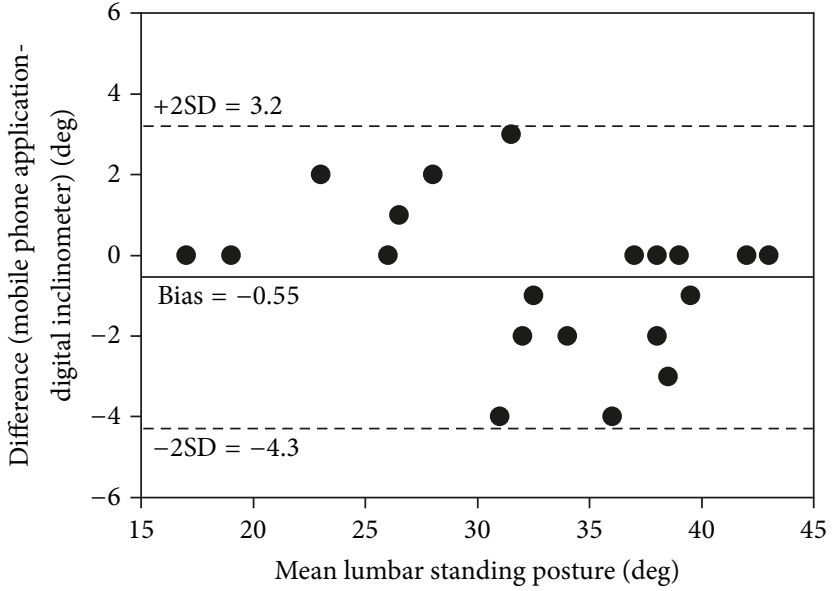

(b)

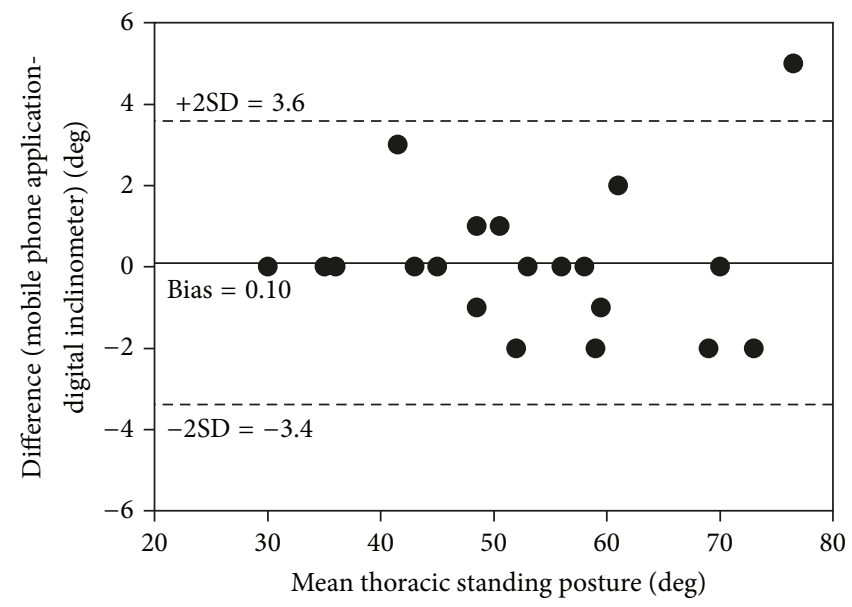

(c)

FIGURE 1: Difference in measures acquired using the IONmed mobile phone application and the digital inclinometer plotted as a function of the mean value for each of the 20 volunteers for (a) lumbosacral angle, (b) lumbar standing posture, and (c) thoracic standing posture. Dotted lines represent the $95 \%$ limits of agreement and solid lines represent the mean difference in measures (bias).

[5]. Therefore, either device provides quick and reliable values characterizing sagittal plane spine curvatures in standing.

In rehabilitation practice, it is important to have a timeefficient method for measuring standing posture in older adults in order to identify hyperkyphosis and associated compensations in the pelvis and lumbar spine indicative of the need for further assessment or to determine the effect of interventions directed at remediating associated impairments and activity limitations. If impairments that contribute to hyperkyphosis cannot be remediated, as in the case of a prevalent vertebral compression fracture, the effect of appropriate multimodel interventions (e.g., medications, body mechanics and posture reeducation, and modification of tasks) can be monitored by tracking stability of the hyperkyphosis. Other causes of age-related hyperkyphosis are modifiable with appropriate interventions. Postural training for hyperkyphotic women using exercises and education has been shown to reduce kyphosis $[7,16]$ and improve balance $[7,17,18]$ and back extensor muscle strength $[7,16]$. These outcomes are particularly important for people at risk of fallrelated and vertebral osteoporotic fractures. Improvements in these physical function domains as well as less restrictions in ADLs were reported in a trial of 108 hyperkyphotic women with a recent osteoporotic spine fracture randomized to an experimental group who wore a type of biofeedbackbased Spinomed orthosis two hours per day for at least six months compared to the wait list control group [19]. Of note, the postural training intervention must be active since passive treatment (taping versus sham taping or no taping) immediately reduced thoracic kyphosis in a group of women with osteoporotic vertebral fractures but did not improve balance or trunk muscle activation [20]. Based on the available evidence, it is recommended that rehabilitation management of people with osteoporosis include postural reeducation and back extensor strengthening $[21,22]$. To evaluate the need for and response to these interventions in the clinical setting, it is critical to have a method that can provide estimates of spine curvatures that are quick and reliable. 
We found a very high level of agreement in the estimates of S, L, and T obtained using the IONmed mobile phone application and the digital inclinometer in people attending an osteoporosis outpatient clinic. Although this method could be used to measure spine curvatures in people regardless of their bone health, it must be remembered that these results are context specific and different results may be obtained in a different patient group. Also, the assessor in this study had seven years of clinical experience using the digital inclinometer to measure standing posture in people at risk of osteoporotic fracture. The same protocol is used for the IONmed mobile phone application method. Different results may be obtained by assessors who have more or less experience using the digital inclinometer to measure spine curvatures. Therefore, others should establish the reliability of the values obtained in their particular clinical setting. We used the IONmed mobile phone application to take the first set of measures in all participants. Balancing the order in which the devices were used would have accounted for potential order effects such as fatigue or learning. However, the Bland-Altman plots confirm that the bias characterizing the difference between the measures obtained with these two devices was small and no systematic error was introduced as a result of this design.

\section{Conclusions}

In our study sample, we found that the IONmed mobile phone application produced estimates for thoracic standing posture, lumbar standing posture, and lumbosacral angle that closely agree with the estimates obtained using the digital inclinometer. The user-friendly application allows the clinician to complete the measurement protocol efficiently with prompts for next steps and automatic recording of measured values. For the growing number of healthcare providers using information technology to facilitate their clinical practice, this mobile phone application offers a quick, convenient method for routine assessment of sagittal spine curvatures.

\section{Conflict of Interests}

Chris P. Recknor invented the IONmed mobile phone application for measuring standing posture (patent pending).

\section{Acknowledgments}

The authors thank the participants who volunteered for this study and Kavin Sutton for collecting these data.

\section{References}

[1] T. Kobayashi, Y. Atsuta, T. Matsuno, and N. Takeda, "A longitudinal study of congruent sagittal spinal alignment in an adult cohort," Spine, vol. 29, no. 6, pp. 671-676, 2004.

[2] A. M. Briggs, J. H. Van Dieën, T. V. Wrigley et al., "Thoracic kyphosis affects spinal loads and trunk muscle force," Physical Therapy, vol. 87, no. 5, pp. 595-607, 2007.
[3] C. Roux, J. Fechtenbaum, S. Kolta, R. Said-Nahal, K. Briot, and C.-L. Benhamou, "Prospective assessment of thoracic kyphosis in postmenopausal women with osteoporosis," Journal of Bone and Mineral Research, vol. 25, no. 2, pp. 362-368, 2010.

[4] A. G. Bruno, D. E. Anderson, J. D’Agostino, and M. L. Bouxsein, "The effect of thoracic kyphosis and sagittal plane alignment on vertebral compressive loading," Journal of Bone and Mineral Research, vol. 27, pp. 2144-2151, 2012.

[5] N. J. MacIntyre, A. L. Lorbergs, and J. D. Adachi, "Inclinometerbased measures of standing posture in older adults with low bone mass are reliable and associated with self-reported, but not performance-based, physical function," Osteoporosis International, vol. 25, pp. 721-728, 2014.

[6] M. Sinaki, E. Itoi, J. W. Rogers, E. J. Bergstralh, and H. W. Wahner, "Correlation of back extensor strength with thoracic kyphosis and lumbar lordosis in estrogen-deficient women," American Journal of Physical Medicine and Rehabilitation, vol. 75, no. 5, pp. 370-374, 1996.

[7] W. B. Katzman, D. E. Sellmeyer, A. L. Stewart, L. Wanek, and K. A. Hamel, "Changes in flexed posture, musculoskeletal impairments, and physical performance after group exercise in community-dwelling older women," Archives of Physical Medicine and Rehabilitation, vol. 88, no. 2, pp. 192-199, 2007.

[8] M. Sinaki, E. Itoi, H. W. Wahner et al., "Stronger back muscles reduce the incidence of vertebral fractures: a prospective 10 year follow-up of postmenopausal women," Bone, vol. 30, no. 6, pp. 836-841, 2002.

[9] N. J. MacIntyre, L. Bennett, A. M. Bonnyman, and P. W. Stratford, "Optimizing reliability of digital inclinometer and flexicurve ruler measures of spine curvatures in postmenopausal women with osteoporosis of the spine: an illustration of the use of generalizability theory," ISRN Rheumatology, vol. 2011, Article ID 571698, 8 pages, 2011.

[10] P. E. Shrout and J. L. Fleiss, "Intraclass correlations: uses in assessing rater reliability," Psychological Bulletin, vol. 86, no. 2, pp. 420-428, 1979.

[11] P. W. Stratford, "Getting more from the literature: estimating the standard error of measurement for reliability studies," Physiotherapy Canada, vol. 56, pp. 27-30, 2004.

[12] J. M. Bland and D. G. Altman, "Statistical methods for assessing agreement between two methods of clinical measurement," The Lancet, vol. 1, no. 8476, pp. 307-310, 1986.

[13] G. J. Fon, M. J. Pitt, and A. C. Thies Jr., "Thoracic kyphosis: range in normal subjects," American Journal of Roentgenology, vol. 134, no. 5, pp. 979-983, 1980.

[14] D. L. Schneider, D. G. Von Mühlen, E. Barrett-Connor, and D. J. Sartoris, "Kyphosis does not equal vertebral fractures: the rancho bernardo study," Journal of Rheumatology, vol. 31, no. 4, pp. 747-752, 2004.

[15] G. A. Greendale, N. S. Nili, M.-H. Huang, L. Seeger, and A. S. Karlamangla, "The reliability and validity of three nonradiological measures of thoracic kyphosis and their relations to the standing radiological Cobb angle," Osteoporosis International, vol. 22, no. 6, pp. 1897-1905, 2011.

[16] E. Itoi and M. Sinaki, "Effect of back-strengthening exercise on posture in healthy women 49 to 65 years of age," Mayo Clinic Proceedings, vol. 69, no. 11, pp. 1054-1059, 1994.

[17] C. Cook, "The relationship between posture and balance disturbances in women with osteoporosis," Physical and Occupational Therapy in Geriatrics, vol. 20, no. 3-4, pp. 37-49, 2002.

[18] P. Suri, D. K. Kiely, S. G. Leveille, W. R. Frontera, and J. F. Bean, "Increased trunk extension endurance is associated 
with meaningful improvement in balance among older adults with mobility problems," Archives of Physical Medicine and Rehabilitation, vol. 92, no. 7, pp. 1038-1043, 2011.

[19] M. Pfeifer, L. Kohlwey, B. Begerow, and H. W. Minne, "Effects of two newly developed spinal orthoses on trunk muscle strength, posture, and quality-of-life in women with postmenopausal osteoporosis: a randomized trial," American Journal of Physical Medicine and Rehabilitation, vol. 90, no. 10, pp. 805-815, 2011.

[20] A. M. Greig, K. L. Bennell, A. M. Briggs, and P. W. Hodges, "Postural taping decreases thoracic kyphosis but does not influence trunk muscle electromyographic activity or balance in women with osteoporosis," Manual Therapy, vol. 13, no. 3, pp. 249-257, 2008.

[21] F. J. Bonner Jr., M. Sinaki, M. Grabois et al., "Health professional's guide to rehabilitation of the patient with osteoporosis," Osteoporosis International, vol. 14, supplement 2, pp. S1-S22, 2003.

[22] L. M. Giangregorio, N. J. MacIntyre, L. Thabane, C. J. Skidmore, and A. Papaioannou, "Exercise for improving outcomes after osteoporotic vertebral fracture," Cochrane Database of Systematic Reviews, no. 7, Article ID CD008618, 2012. 


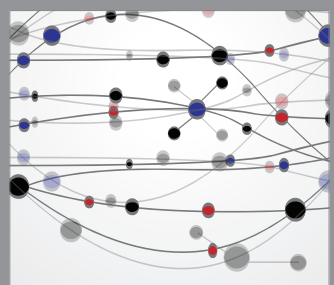

The Scientific World Journal
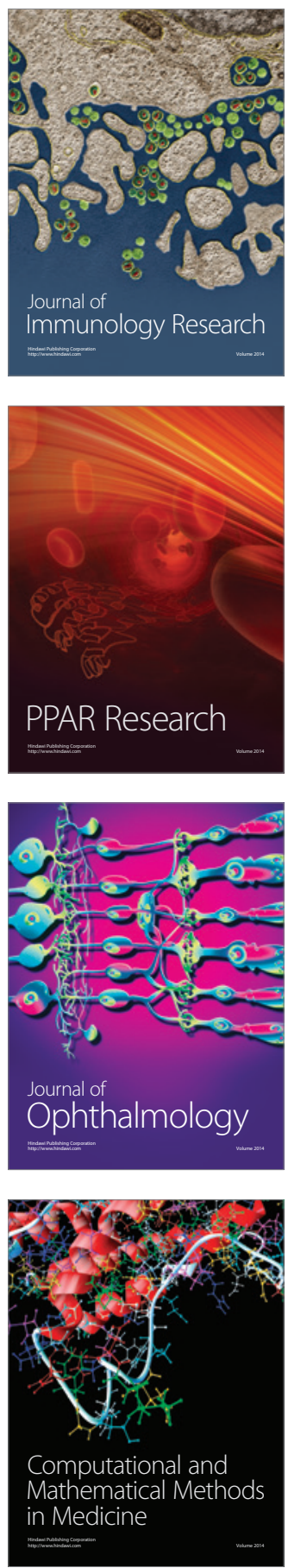

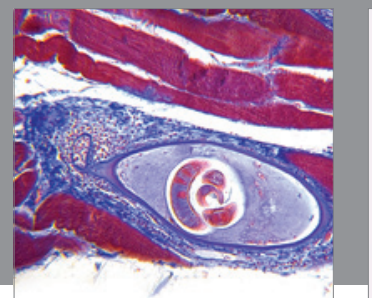

Gastroenterology

Research and Practice
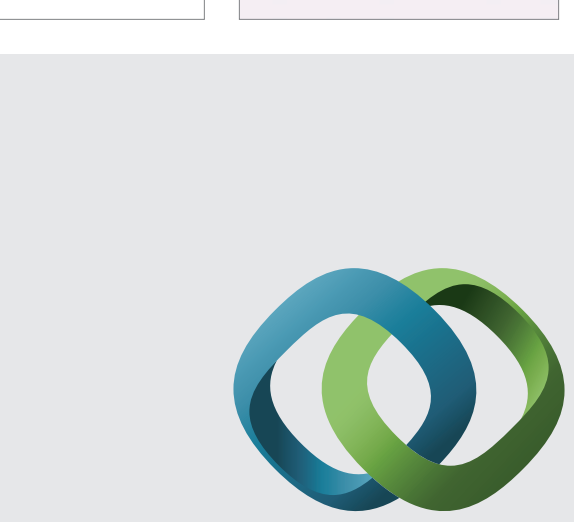

\section{Hindawi}

Submit your manuscripts at

http://www.hindawi.com
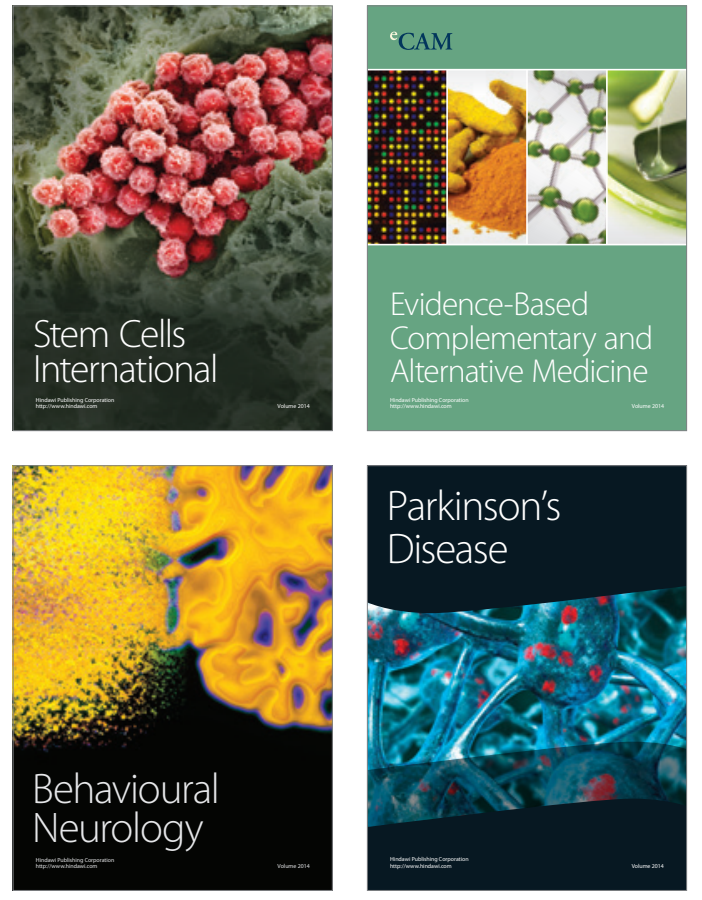
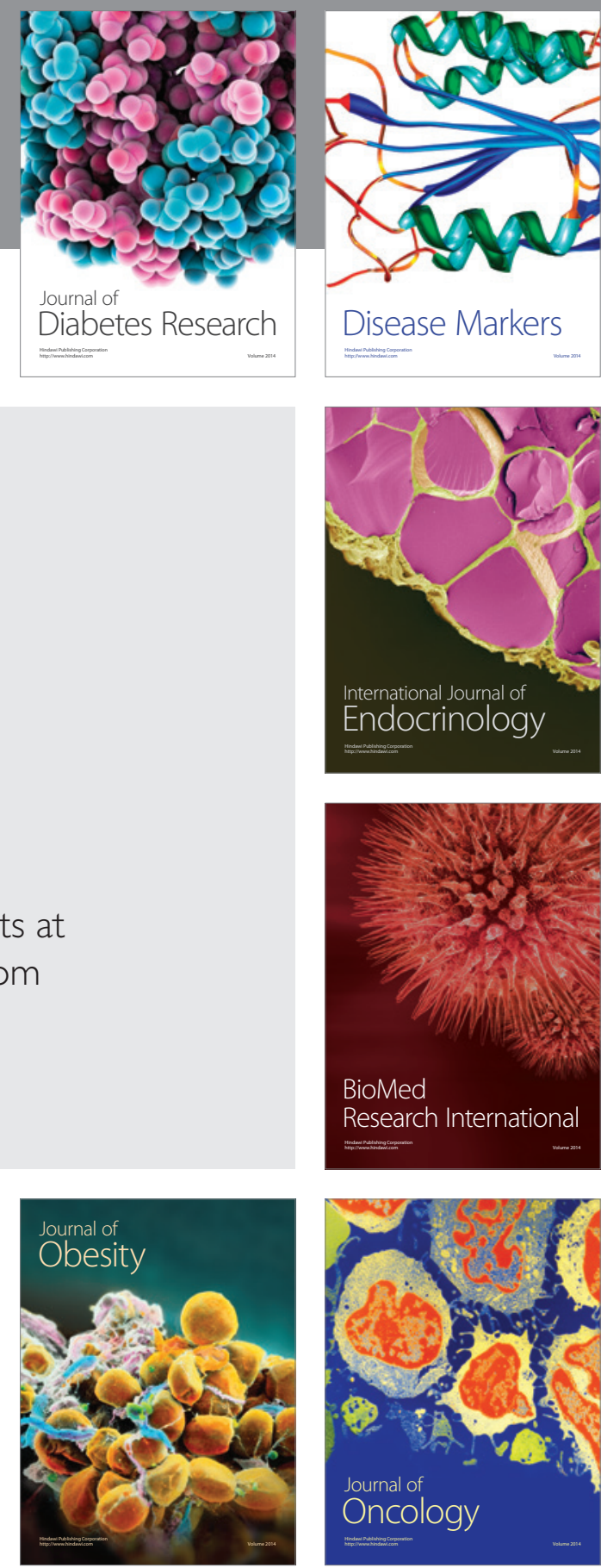

Disease Markers
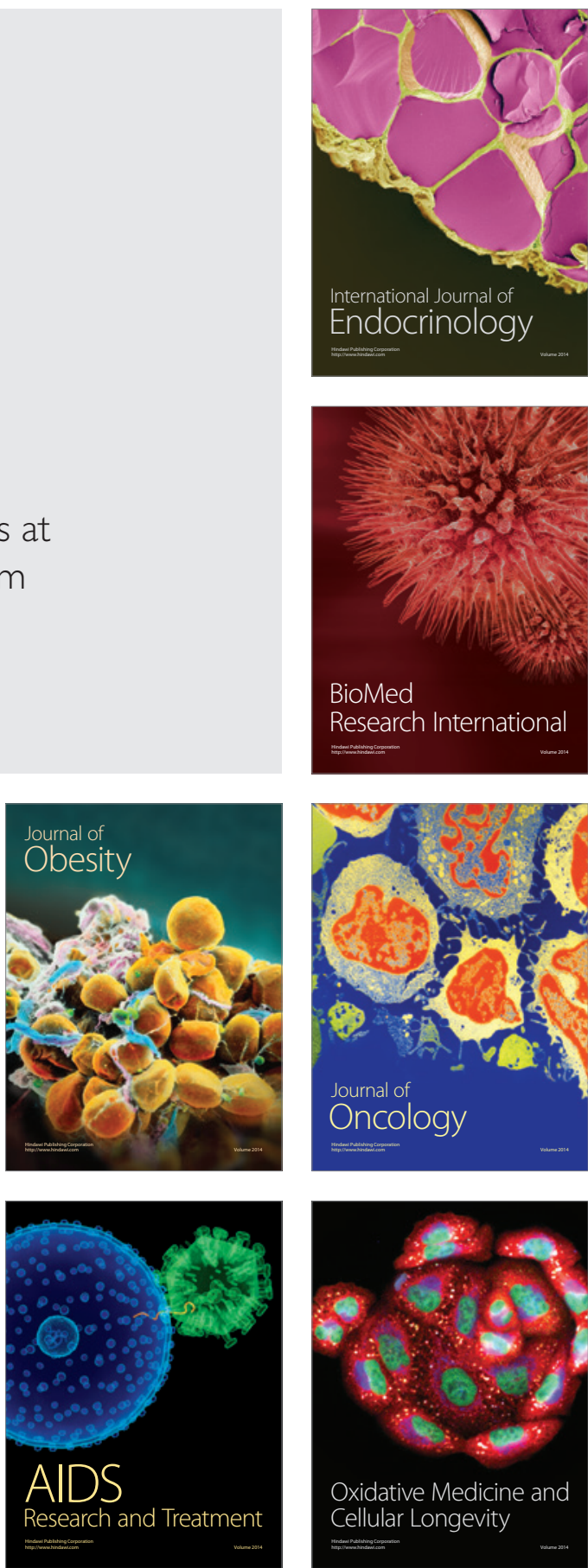